\title{
A field based assessment of the aerodynamics of operational freight trains
}

\author{
A. Quazi ${ }^{1}$, T. Crouch $^{1}$, M. C. Thompson ${ }^{1}$, T. McGreevy ${ }^{2}$, J. Bell ${ }^{3}$, D. Burton ${ }^{1}$ \\ ${ }^{1}$ Department of Mechanical and Aerospace Engineering, Monash University, Melbourne, Victoria, 3800, Australia \\ 2 Pacific National, Melbourne, Victoria, 3003, Australia \\ ${ }^{3}$ German Aerospace Center (DLR), Institute for Aerodynamics and Flow Technology (AS), \\ Göttingen, Lower Saxony, 37073, Germany
}

\begin{abstract}
A novel field based method for analysing the aerodynamic pressure drag acting on intermodal freight trains is presented. A full-scale $48 \mathrm{ft}$ shipping container was instrumented with surface pressure taps and loaded onto a number of single and doublestacked container freight trains that operate between Melbourne and Perth, Australia (a distance of 3,500km). Container surface pressure data enabled the instrumented container pressure drag coefficient to be evaluated for a range of different train loading configurations, container positions along the train and atmospheric wind conditions. Field based measurements show that for low wind conditions surface pressure distributions measured on the front and base faces of the instrumented container are synonymous with those reported on in past studies. However, the magnitude of the pressure drag coefficient was found to be approximately $50 \%$ lower for all loading cases analysed compared with previous analogous wind-tunnel and numerical investigations. For high wind conditions the drag coefficient was generally found to increase and this change in the drag coefficient was well correlated with the level of asymmetry observed in the measured pressure distributions. In the absence of direct information of the incident free-stream wind conditions, the level of asymmetry in the pressure distributions is found to provide a viable indirect method for assessing the impact that cross-winds have on the aerodynamics of freight trains.
\end{abstract}

\section{Keywords}

Aerodynamics; freight trains; full-scale experiments; field study; surface pressure.

\section{Introduction}

Diesel locomotives on freight trains are the most common type of locomotives used in the transport of large quantities of goods over long distances. Such freight trains can be over $1.8 \mathrm{~km}$ in length and can travel in excess of $110 \mathrm{~km} / \mathrm{h}$, with the potential of faster speeds in the future, including within Australia where transcontinental journey are frequent. Owing to the long and bluff body geometry of freight trains, which can have length to height ratios $(L / H)$ between $250-500$, the aerodynamic drag component is of significance.

Despite the importance of the aerodynamic resistive forces on emissions and fuel efficiency, the aerodynamics of freight trains has not received the same level of research focus when compared to other vehicles, such as cars and high speed trains. Aerodynamic flows around freight trains are complex, owing to the fact that the rolling stock can vary in type, length and height (e.g., flat and well wagons); containers are of varying size, construction and can be single or double-stacked; and empty slots may exist. Typically, aerodynamic considerations are not prioritised when loading freight trains. Rather, freight trains are often loaded according to weight distribution and volume efficiency [1]. Previous studies indicate that by optimising the loading configuration of shipping containers, through minimis- ing the spacing between adjacent containers, a 27\% decrease in the overall resistance can be achieved hence reducing the fuel consumption and emitted emissions. [1].

To date our current understanding of the aerodynamics loading of freight trains has largely been derived through windtunnel [2], moving-model [5], and computational fluid dynamics (CFD) methods [3]. Due to the very large length scales and Reynolds numbers involved, a major limitation of utilising these techniques is the ability to accurately simulate and predict the flow field for operational conditions. To gain confidence in the ability to fully realise the aerodynamic performance gains predicted by these studies field based assessments are required.

In this study we present aerodynamic pressure drag data obtained from measurements performed on a number of single and double-stacked operational freight trains. This is significant as it enables the variation in the train aerodynamic drag to be assessed as a function of distance along the train for real loading configurations, operational Reynolds numbers and environmental wind conditions.

\section{Methodology}

Figure 1 illustrates the route taken by the freight trains where it departed from Melbourne travelling to Perth stopping via Adelaide and returned back to Melbourne. The front and base surfaces of the container were fitted with a total of 118 surface pressure tapes. Figure 2 shows the container on-board an operational train along with the array of pressure taps on the front face. Surface pressure data was logged using an on-board Data Acquisition System over the entire journey at a frequency of $1 \mathrm{~Hz}$. A rolling 60 second time-average filter with no overlap was applied to this data set. Findings presented in this study are a result of excluding data that was measure when the train ground speed was below $60 \mathrm{~km} / \mathrm{h}$. An on-board Global Positioning System (GPS) provided the train velocity, altitude and position information throughout the route. A total of 15 double and single-stacked trains were involved in this study. Figure 3 illustrates the different stacking types that were tested and location of the instrumented container along the train.

The pressure drag coefficient, $C_{D, p}$, was determined by integrating pressure distributions to give an area averaged surface pressure over the front, $\bar{P}_{A, \text { front }}$, and base, $\bar{P}_{A, \text { base }}$, container surfaces,

$$
C_{D, p}=\frac{\bar{P}_{A, \text { front }}-\bar{P}_{A, \text { base }}}{1 / 2 \cdot \rho \cdot V_{\text {train }}^{2}},
$$

where the reference dynamic pressure is dependent on the measured train ground speed, $V_{\text {train }}$, obtained from GPS data, and the air density, $\rho$, is determined from near by weather station data sources. As the primary concern is the influence that gap size has on aerodynamic performance, we refer to the aerody- 


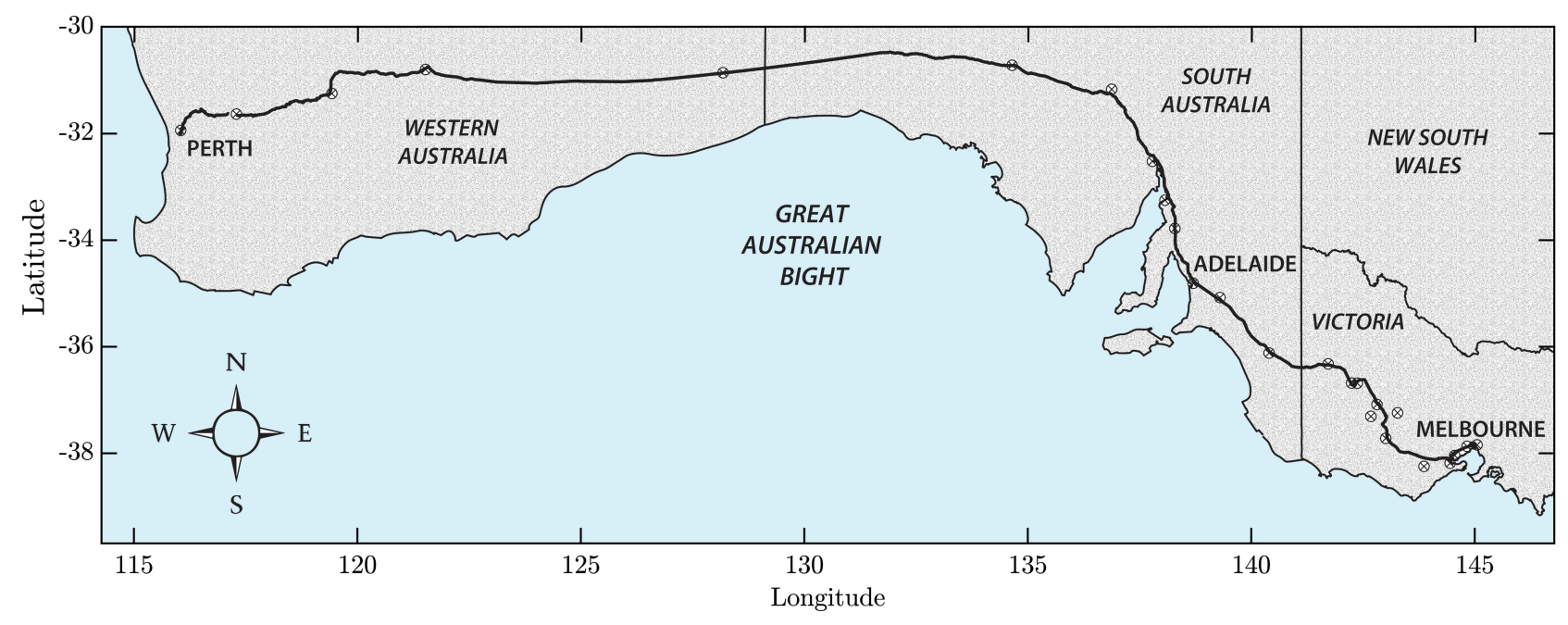

Figure 1. Path taken by the train starting in Melbourne and finishing at Perth. The $\otimes$ markers are weather stations.
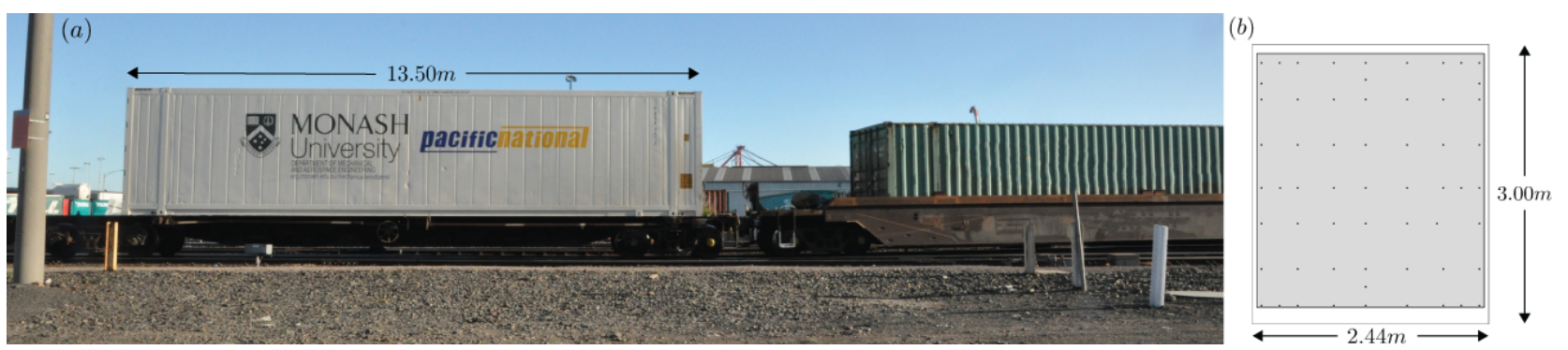

Figure 2. (a) Instrumented $48 \mathrm{ft}$ refrigeration shipping container (white) on board an operational freight train. (b) Array of pressure taps on the front face where the shaded area represents the region over which pressure distributions have been interpolated.

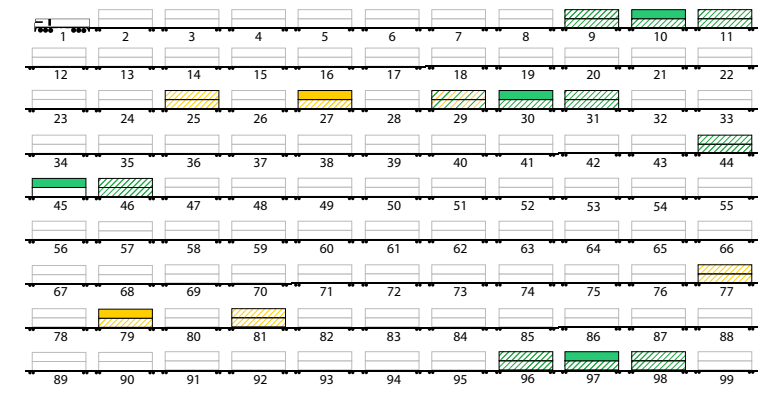

(a) double-stacked gap sizes. $\square: 1.75 \mathrm{~W}-3.37 \mathrm{~W}, \square: 11.43 \mathrm{~W}-12.10 \mathrm{~W}$

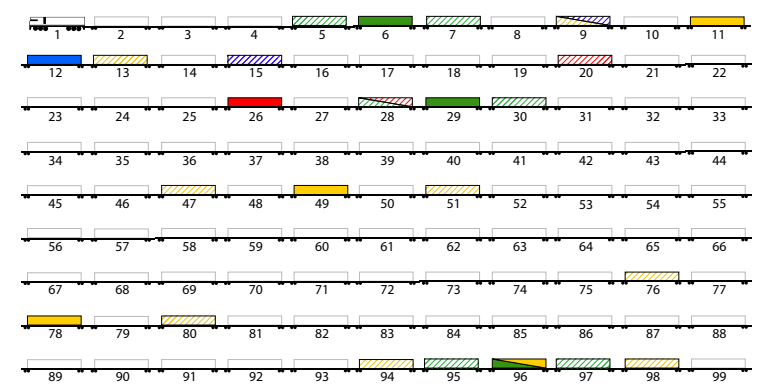

(b) single-stacked gap sizes. $\square: 0.26 \mathrm{~W}-0.76 \mathrm{~W}, \square: 4.97 \mathrm{~W}-6.87 \mathrm{~W}$ $13.57 \mathrm{~W}, \mathrm{a}: 21.88 \mathrm{~W}$

Figure 3. (a) Double and (b) single stacked configurations that were tested. Note only the local loading configuration either side of the instrumented container is shown. namic drag acting on a gap as opposed to the drag acting on the container. Here we define the non-dimensional gap size as the ratio of the size of the gap, $G$, to the width of the container, $W$, We note that for all test cases, except for the largest gap size test case, the gap size immediately upstream and downstream of the container is the same. Meaning the pressure drag acting on the container is equivalent to the pressure force that acts on the upstream or downstream gap size. This approach is based on the assumption that front pressures acting on the container are primarily dependent on the upstream gap size and the base pressure is primarily a function of the base gap size, which has been demonstrated by Li et al. [2] and Maleki et al. [3].

In addition to on-board train measurements, weather station data was obtained from the Australian Bureau of Meteorology. Weather stations selected for this study were located within $13 \mathrm{~km}$ to $32 \mathrm{~km}$ of the train line and recorded the mean sea level pressure, temperature, wind speed and direction. The location of these weather stations are shown in figure 1 . The air density was calculated using this data and corrected for the altitude. Based on the proximity of the instrumented container to the weather stations, which numbered 26 in total, wind speed and direction data (corrected to a height of $3 \mathrm{~m}$ ) is used to classify the wind environment during testing into low and high wind conditions. Low wind conditions correspond to weather station data recording a wind speed of $\leq 5 \mathrm{~km} / \mathrm{h}$ and high wind conditions being $\geq 5 \mathrm{~km} / \mathrm{h}$. 

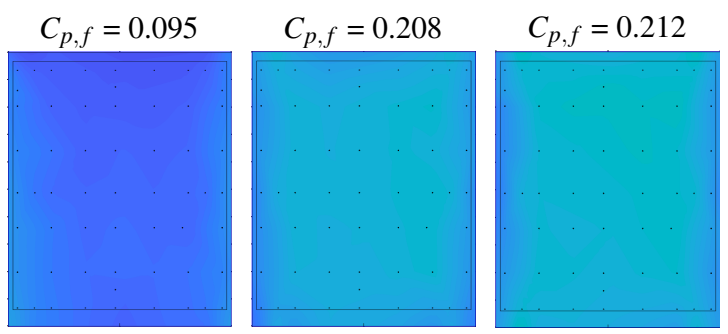

(a) $\mathrm{G}=1.75 W$

(b) $\mathrm{G}=3.37 \mathrm{~W}$

(c) $\mathrm{G}=3.37 \mathrm{~W}$
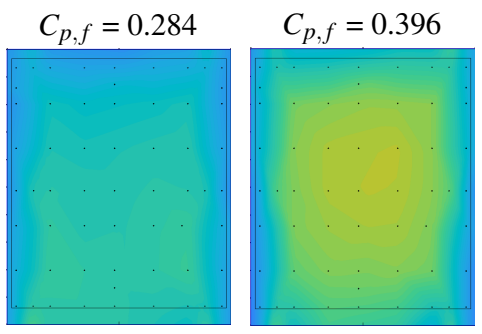

(d) $\mathrm{G}=3.87 \mathrm{~W}$

(e) $\mathrm{G}=11.43 W$

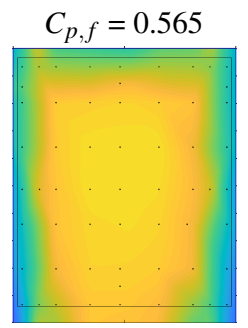

(f) $\mathrm{G}=12.10 \mathrm{~W}$

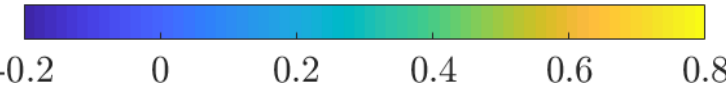

Figure 4. Front surface pressure contour plots for gap sizes ranging from $1.75 \mathrm{~W}$ to $12.10 \mathrm{~W}$ for test cases when the container was positioned on top of a double-stacked loading configuration.

\section{Results and discussion}

\section{Low wind results}

For low wind conditions figure 4 illustrates pressure contours and magnitudes of the container front surface $\left(C_{p, f}\right)$ for varying gap sizes for cases when the instrumented container was loaded on top of a double-stacked loading configuration. The contours exhibited similar behaviours to that seen by Li et al. [2], where low pressures are observed towards the edges as the air accelerates around the corner and a high stagnation pressure region exists at the centre. Figures $4 b, 4 c, 4 d$ all exhibit a similar pressure magnitude and distribution for a similar gap size despite the difference in location of each test case $(1400 \mathrm{~m}, 140 \mathrm{~m}$ and $440 \mathrm{~m}$ respectively) from the nose of the train. This indicates that the primary effect on container surface pressures may be a result of the local gap size with the position along the train having a much lower order effect after a certain distance from the nose of the train.

For both single and double-stacked containers figure 5 highlights the variations in the gap pressure drag coefficient as a function of the gap size, $G$. The error bars represent the range of pressure drag coefficients measured during high wind conditions. Both the magnitude and the change in the pressure drag coefficient with gap size are very similar for both the single and double-stacked container cases. The drag coefficient is observed to increase rapidly from a gap size of $1.8 \mathrm{~W}$ up to approximately $4.0 \mathrm{~W}$. Once the gap size exceeded $14.0 \mathrm{~W}$ significant increases in the pressure drag coefficient are no longer seen.

Also included in figure 5 are results from Li et al. [2] and Maleki et al. [3] who performed wind-tunnel and numerical investigations on a simplified double-stacked train over a range of gap sizes respectively. At the smallest single-stacked gap sizes the field results compare well with these studies. However, for gap sizes $\geq 1.75 \mathrm{~W}$ the field results are approximately $50 \%$ lower

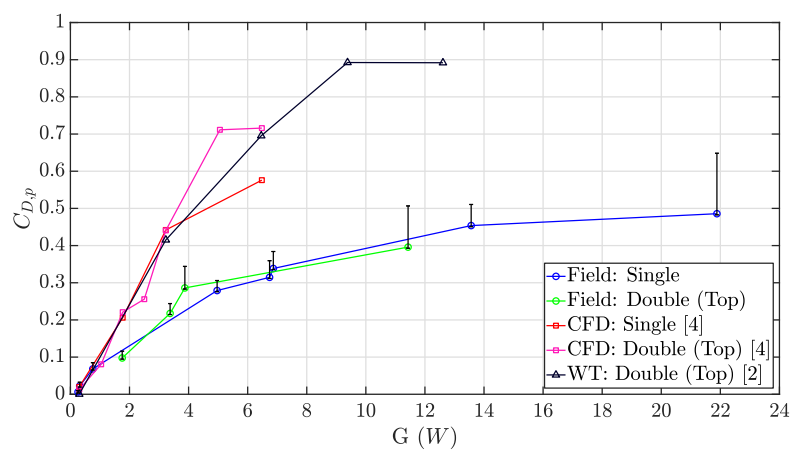

Figure 5. A comparison of change in drag coefficient with gap size for single and double-stacked configurations between this field study and investigations of Li et al. [2] and Maleki et al. [3].

than those reported in the wind-tunnel and numerical investigations. One of the primary differences between these studies is the field results have been obtained at positions $\geq 140 \mathrm{~m}$ from the nose of the train. The studies of Li et al. [2] and Maleki et al. [3], were carried out at distances between $46 \mathrm{~m}-75 \mathrm{~m}$ at full scale. It is hypothesised that the large variation in the position along the train at which drag coefficients have been assessed, is the primary reason for the difference in drag coefficients reported between the studies.

\section{High wind results}

Throughout the duration of field experiments the instrumented container was exposed to a wide range of environmental wind conditions. As indicated by the error bars (range bars) of figure 5, compared to the low wind test cases the high wind cases generally resulted in an increase in aerodynamic pressure drag. One of the defining characteristics of the high wind cases compared to the low wind results was the level of asymmetry observed in the measured front surface pressure distributions. Examples of this distribution for selected stacking configurations are highlighted in figure 6 . To further understand how this distribution effects the drag coefficient an asymmetry measure,

$$
C_{P_{\alpha}}=C_{P_{\text {front }, \text { left }}}-C_{P_{\text {front }, \text { right }}},
$$

was defined as the absolute difference of the left and right halve area averaged pressure coefficients.

Figure 7 plots the pressure drag coefficient with the asymmetry measure of the front surface pressure distributions $\left(C_{P_{\alpha}}\right)$. A least squares quadratic line of best fit has been applied to each of the test cases. The dash-dot line represents a single-stacked train with one empty slot either side of the container $(\sim 6.7 \mathrm{~W})$. The dashed line represents a double-stacked configuration, with the instrumented container load on top, with one empty slot either side $(\sim 11.8 W)$. The dotted line is a double-stacked container configuration with no empty slot either side $(\sim 3.5 W)$. Despite the position of the container along the train for each configuration being anywhere between $140 \mathrm{~m}$ to $1400 \mathrm{~m}$, for similar stacking configurations the quadratic fits are reasonably consistent. Asymmetry levels $\leq 0.05$ are consistent with nearby weather station data recording low wind conditions. Asymmetry levels $\geq 0.05$ were generally found to correspond to high wind conditions where the prevailing wind direction would result in the train experiencing a cross-wind. Further to this in figure $4 \mathrm{e}$ and figure $4 \mathrm{f}$ the pressure distributions are symmetric suggesting a low wind conditions, however, the difference in the pressure coefficient is $\sim 0.17$. This highlights that only considering the asymmetry level is not sufficient and assessing the 

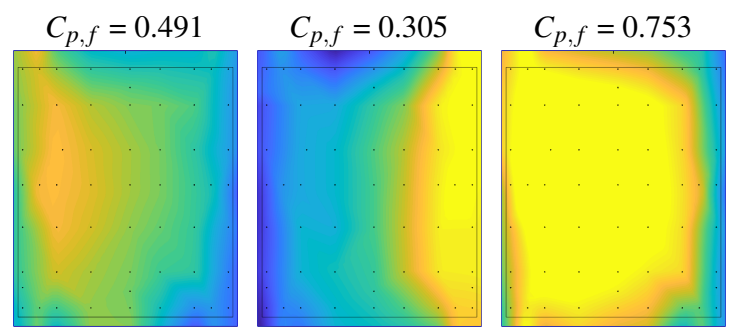

(a) $\mathrm{G}=6.7 \mathrm{~W}$ (Single)

(b) $\mathrm{G}=3.5 \mathrm{~W}$ (Double)

(c) $\mathrm{G}=11.8 \mathrm{~W}$ (Double)

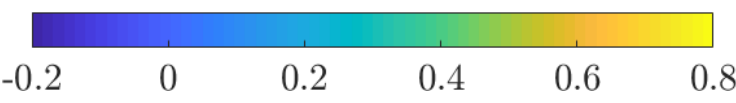

Figure 6. Asymmetric distribution of front surface pressure contours plots for selected stacking configurations.

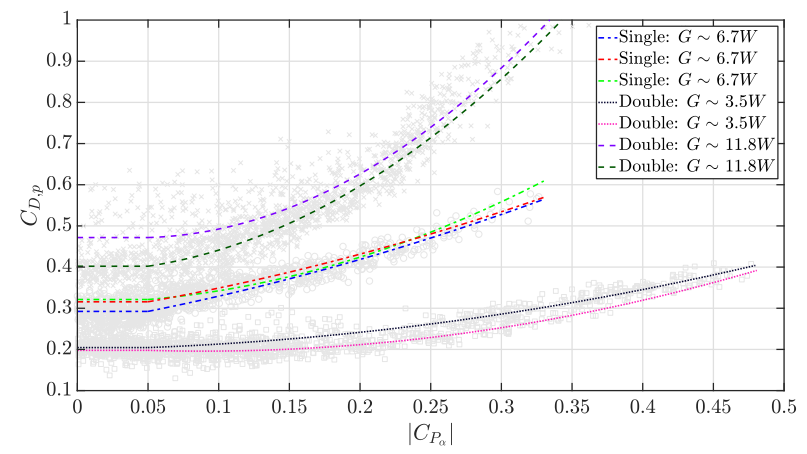

Figure 7. Change in drag coefficient with the asymmetry criterion for different stacking configurations.

wind speed and direction of nearby weather stations is critical to validate observations made in the pressure distributions. For the trip associated with the stacking configuration in figure $4 \mathrm{e}$ a significant head wind of $\sim 35 \mathrm{~km} / \mathrm{h}$ was found.

Between the three different loading configurations analysed in figure 7 we see distinct differences in the general nature of the trend lines. Comparing the double-stacked container cases, the larger gap size $(\sim 11.8 \mathrm{~W})$ leads to a higher drag coefficient at lower asymmetry levels and a higher rate of change in drag compared to the smaller gap size case $(\sim 3.5 \mathrm{~W})$. The larger gap size data also has a higher standard deviation highlighted by the spread in the data and has a relatively large offset between the trend line for the two repeated trips at low asymmetry values and relates directly to the front pressure distribution seen in Figures $4 \mathrm{e}$ and $4 \mathrm{f}$. This finding is consistent with pressures acting on containers with larger upstream gaps being more susceptible to changes in the magnitude of head and tail winds. As the asymmetry increases for this case the trend lines become increasing similar. For the smaller gap sizes the pressure drag coefficient is less sensitive to changes in the level of asymmetry compared to large gap sizes. This is in-line with Soper et al. [5] and Maleki et al. [4] who also found similar results over different gap sizes for a given a yaw angle.

\section{Conclusion}

A novel field study was employed where a full-scale shipping container was pressure tapped on the front and base surfaces and placed on a number operational freight trains. This work represents a rare field study that demonstrates an empirical method for determining surface pressure distributions and pressure drag on a train container under natural environmental conditions. For all gap sizes and stacking configurations tested the pressure drag coefficient during low wind conditions was found to be $50 \%$ lower than past wind-tunnel and numerical studies. However, the surface pressure distribution and the relative trend with increasing gap size was seen to be similar.

Throughout the train journey periods of high asymmetry in the frontal surface pressure distributions were found to be consistent with cross-wind conditions and higher pressure drag coefficients. An asymmetry measure was established, as a proxy for the wind yaw angle, and a quadratic relationship was drawn between the pressure drag coefficient and this measure. Larger gap sizes corresponded to a higher pressure drag and also a higher rate of change with the asymmetry measure. For smaller gap sizes high levels of asymmetry was found to correlate to smaller changes in the drag coefficient.

The consistency in the relationships drawn here for a range of stacking configurations over multiple trips provides confidence that the experimental field techniques described herein are a viable method to assess the aerodynamic performance of fullscale operational freight trains for a large range of operating conditions. It is acknowledged that further work is required to develop a relationship between the asymmetry levels and the real wind yaw angles that the container experiences. This can be achieved through further wind-tunnel or numerical based studies where the yaw angle is known and can be empirically related to observed asymmetry levels.

\section{Acknowledgements}

This research was supported through Pacific National Rail and the Australian Research Council's Linkage Project funding scheme, under project number LP13100953.

\section{References}

[1] Lai, Y.-C., Barkan, C. P. and Önal, H., Optimizing the aerodynamic efficiency of intermodal freight trains, Transportation Research Part E: Logistics and Transportation Review, 44, 2008, 820-834.

[2] Li, C., Burton, D., Kost, M., Sheridan, J. and Thompson, M. C., Flow topology of a container train wagon subjected to varying local loading configurations, Journal of Wind Engineering and Industrial Aerodynamics, 169, 2017, 1229.

[3] Maleki, S., Burton, D. and Thompson, M. C., Flow structure between freight train containers with implications for aerodynamic drag, Journal of Wind Engineering and Industrial Aerodynamics, 188, 2019, 194-206.

[4] Maleki, S., Burton, D. and Thompson, M. C., On the flow past and forces on double-stacked wagons within a freight train under cross-wind, Journal of Wind Engineering and Industrial Aerodynamics, in print.

[5] Soper, D., Baker, C. and Sterling, M., An experimental investigation to assess the influence of container loading configuration on the effects of a crosswind on a container freight train, Journal of Wind Engineering and Industrial Aerodynamics, 145, 2015, 304-317. 